\title{
Diagnosing Vasospasm After Subarachnoid Hemorrhage: CTA and CTP
}

\author{
Craig D. Wilson, Jai Jai Shiva Shankar
}

\begin{abstract}
Cerebral vasospasm is a potentially devastating complication in patients with aneurysmal subarachnoid hemorrhage. The purpose of this article is to review the use of computed tomogram (CT) angiography and CT perfusion in the diagnosis of cerebral vasospasm after aneurysmal subarachnoid hemorrhage and also assess their use in guiding treatment decisions. Both techniques are widely used for other indications but their use in cerebral vasospasm has not been well defined. Computed tomogram angiography can directly visualize arterial narrowing and CT perfusion is able to evaluate differences in perfusion parameters after aneurysmal subarachnoid hemorrhage with high sensitivity and specificity. $\mathrm{CT}$ perfusion is better at predicting which patients require endovascular treatment.
\end{abstract}

RÉSUMÉ: Diagnostic du vasospasme après une hémorragie sous-arachnoïdienne : CTA et CTP. Le vasospasme cérébral est une complication potentiellement dévastatrice chez les patients atteints d'une hémorragie sous-arachnoïdienne due à un anévrisme. Le but de cet article est de revoir l'utilisation de l'angiographie par tomodensitométrie et de la tomodensitométrie de perfusion dans le diagnostic du vasospasme cérébral après une hémorragie sous-arachnoïdienne anévrismale et d'évaluer leur utilité dans la prise de décision concernant le traitement. Ces deux techniques sont largement utilisées pour d'autres indications, mais leur utilisation pour le diagnostic du vasospasme cérébral n'est pas très bien définie. L'angiographie par tomodensitométrie peut visualiser directement un rétrécissement artériel et le CT de perfusion peut évaluer des différences dans les paramètres de perfusion après une hémorragie sous-arachnoïdienne anévrismale avec un haut degré de sensibilité et de spécificité. Le CT de perfusion est meilleur pour prédire quels patients nécessitent un traitement endovasculaire.

Can J Neurol Sci. 2014; 41: 314-319

Aneurysmal subarachnoid hemorrhage (A-SAH) has potentially devastating consequences acutely, sub-acutely and long term. The incidence of A-SAH varies widely among populations with up to a tenfold difference. Incidence ranges from 2.0 per 100,000 per year in China to 22.5 per 100,000 per year in Finland. ${ }^{1}$ One of the most feared complications in patients who survive the acute A-SAH is cerebral vasospasm (CV). The peak incidence of CV is between 5 and 14 days after A-SAH. Cerebral vasospasm may occur angiographically in up to two-thirds of A-SAH patients, however this is only symptomatic in about one-third of them. Delayed neurologic defects or delayed cerebral ischemia (DCI) occurs in approximately half of patients with angiographic CV., Early diagnosis and treatment of $\mathrm{CV}$ can reduce morbidity and mortality. ${ }^{4}$ Therefore, it is important to diagnose and thereafter treat $\mathrm{CV}$ as early as possible in order to avoid permanent neurologic deficit.

The pathogenesis of CV is a poorly understood, although it can be thought of as an abnormal and prolonged contraction of vascular smooth muscle due to the presence of subarachnoid blood. Breakdown products of blood in the subarachnoid space appear to be involved in the development of CV after SAH. In a canine model, a relationship between the volume of blood in the subarachnoid space and the severity of CV was demonstrated. ${ }^{5}$ In humans, the amount of blood on computed tomogram (CT) in patients with $\mathrm{SAH}$ has been shown to correlate with the severity of $\mathrm{CV}^{6}$

There is some evidence that oxyhemoglobin may be the primary blood product causing CV. Oxyhemoglobin has been shown to be present in high concentrations in cerebrospinal fluid during $\mathrm{CV}$ and has been shown to be the most vasoactive substance within blood. ${ }^{7}$ There is also some evidence that free radicals play a role in the pathogenesis of $\mathrm{CV}$, in that antioxidants have shown some ability to improve vasospasm. ${ }^{2}$

\section{DEFINITIONS}

There is considerable inconsistency and overlap in terminology surrounding cerebral vasospasm and delayed cerebral ischemia. Some authors use the term CV to refer to the clinical finding of delayed onset neurologic deficit after A-SAH and some use the term purely to refer to radiographic evidence

From the Department of Diagnostic Radiology (CDW), Department of Neuroradiology (JJSS), Dalhousie University, Halifax, Nova Scotia, Canada.

Received August 30, 2013. Final Revisions Submitted November 20, 2013. Correspondence to: Jai Jai Shiva Shankar, Department of Neuroradiology, Dalhousie University, Halifax, Nova Scotia, B3K 6A3, Canada. Email: shivajai1@gmail.com. 
of vessel narrowing. Relatively recently, a multidisciplinary expert panel recommended that future clinical trials and observational studies use a uniform definition of these terms to make future comparison among studies easier. The term DCI should refer to two main outcomes: [1] new neurologic deficit that was not present immediately after aneurysm occlusion and cannot be attributed to other causes, and [2] cerebral infarction on computed tomogram $(\mathrm{CT})$ or magnetic resonance imaging (MR) that was not present immediately after aneurysm occlusion and not attributable to other causes. They recommend that the use of the words "vasospasm" or "arterial narrowing" be restricted to descriptions of a radiological test. ${ }^{8}$

The terms CV and DCI are related. Delayed cerebral ischemia is often the consequence of CV and CV is often the cause of DCI. The goal is to diagnose CV early enough and accurately enough to initiate appropriate therapy and avoid progression to DCI.

\section{Modalities for Assessment of Vasospasm}

Digital subtraction angiography (DSA) is considered the gold standard method for the assessment of CV. Other methods that have been used in the evaluation of $\mathrm{CV}$ include transcranial Doppler ultrasound, CT angiography (CTA), CT perfusion (CTP) and MRI. The following sections will review the use of CTA and CTP in the diagnosis of vasospasm and/or DCI.

\section{CT Angiography in the Diagnosis of Vasospasm}

Computed tomogram angiography is a non-invasive technique which, compared to DSA, requires considerably less manpower and is much more readily available. As with DSA, CTA is able to directly visualize arterial narrowing related to cerebral vasospasm. There have been several studies published assessing the diagnostic potential of CTA for CV after A-SAH. The data is summarized in the Table. Figures $1 \mathrm{a}$ and $1 \mathrm{~b}$ demonstrate the CTA findings of CV.

A study by Anderson et al compared CTA with DSA and found high sensitivity and specificity for CTA in the detection of $\mathrm{CV}$. This was particularly evident in cases where there was no $\mathrm{CV}$, with sensitivity of $92 \%$ and specificity of $80 \%$, as well as in cases of severe $\mathrm{CV}$, with sensitivity of $100 \%$ and specificity of $100 \% .{ }^{9}$ Goldsher et al specifically looked at the use of CTA in diagnosing CV involving the vertebrobasilar system and showed that CTA was also accurate in this situation. ${ }^{10}$ Wintermark et al also compared CTA with DSA and found a slightly lower sensitivity for CTA detection of $\mathrm{CV}$ of $75.6 \%$, but maintained a high specificity of $95.3 \% .{ }^{11}$ Yoon et al found a very high sensitivity and specificity of 97.5 and $98.1 \%$ respectively when comparing CTA with DSA. ${ }^{12}$ Binaghi et al had similar results comparing CTA with DSA finding sensitivity and specificity of $87.7 \%$ and $99.2 \%$ respectively. ${ }^{13}$ Chaudhary et al prospectively compared CTA with DSA and demonstrated sensitivity of $63 \%$ and specificity of $90 \%$ for CV. ${ }^{14}$

Several of the above-mentioned studies were included in the meta-analysis by Greenberg et al that estimated $79.6 \%$ sensitivity and $93.1 \%$ specificity for the diagnosis of CV using CTA. They were able to perform a sub-analysis on several of the papers that included data on proximal and distal segments. Proximal segments were considered to be the internal carotid artery (ICA), the A1 segment of the anterior cerebral artery, the M1 segment of the middle cerebral artery, the anterior communicating artery, the vertebral artery, the basilar artery, the P1 segment of the posterior cerebral artery, and the posterior communicating artery. The pooled estimates for proximal vasospasm found an $81.7 \%$ sensitivity and $93.7 \%$ specificity.

Table: Summary of CTA and CTP studies which investigated the diagnosis of CV or DCI

\begin{tabular}{|c|c|c|c|c|c|c|c|}
\hline Imaging Modality & Authors & $\mathrm{N}$ patients & Diagnosis & Gold Standard & CT Scanner & Sensitivity (\%) & Specificity (\%) \\
\hline CTA & Anderson et al. & 17 & $\mathrm{CV}$ & DSA & Not reported & $57-100$ & $80-100$ \\
\hline CTA & Goldsher et al. & 36 & CV & $\begin{array}{c}\text { Transcranial } \\
\text { Doppler }\end{array}$ & $\begin{array}{c}\text { Phillips MX } 8000 \\
\text { (16 slice) }\end{array}$ & Not reported & Not Reported \\
\hline CTA & Wintermark et al. & 27 & $\mathrm{CV}$ & DSA & 8 or 16 slice & 75.6 & 95.3 \\
\hline CTA & Yoon et al. & 17 & CV & DSA & $\begin{array}{c}\text { Phillips MX } 8000 \\
\text { (16 slice) }\end{array}$ & 97.5 & 98.1 \\
\hline CTA & Binaghi et al. & 22 & $\mathrm{CV}$ & DSA & 16 slice & 87.7 & 99.2 \\
\hline CTA & Chaudhary et al. & 33 & $\mathrm{CV}$ & DSA & 16 slice & 63 & 90 \\
\hline CTA & Shankar et al. & 34 & CV & DSA & $\begin{array}{c}\text { Toshiba Aquilion } \\
64 \text { (64 slice) }\end{array}$ & $82-92$ & $50-90$ \\
\hline CTA & Dankbaar et al. & 39 & $\mathrm{DCl}$ & Clinical (DCI) & $\begin{array}{l}\text { Philips Mx8000 } \\
\text { LDT (64 slice) }\end{array}$ & 64 & 50 \\
\hline СТP & Binaghi et al. & 23 & CV & DSA & 16 slice & $20-90$ & 100 \\
\hline СТP & Dankbaar et al. & 39 & $\mathrm{DCl}$ & Clinical (DCI) & $\begin{array}{l}\text { Philips Mx8000 } \\
\text { LDT (64 slice) }\end{array}$ & 84 & 79 \\
\hline CTP & Moftakhar et al. & 14 & CV & DSA & GE 8 or 16 slice & Not reported & Not reported \\
\hline CTP & Killeen et al. & 57 & $\mathrm{DCl}$ & Clinical (DCl) & $\begin{array}{l}\text { GE Lightspeed or } \\
\text { Pro-16 (16 Slice) }\end{array}$ & 80 & 67 \\
\hline
\end{tabular}




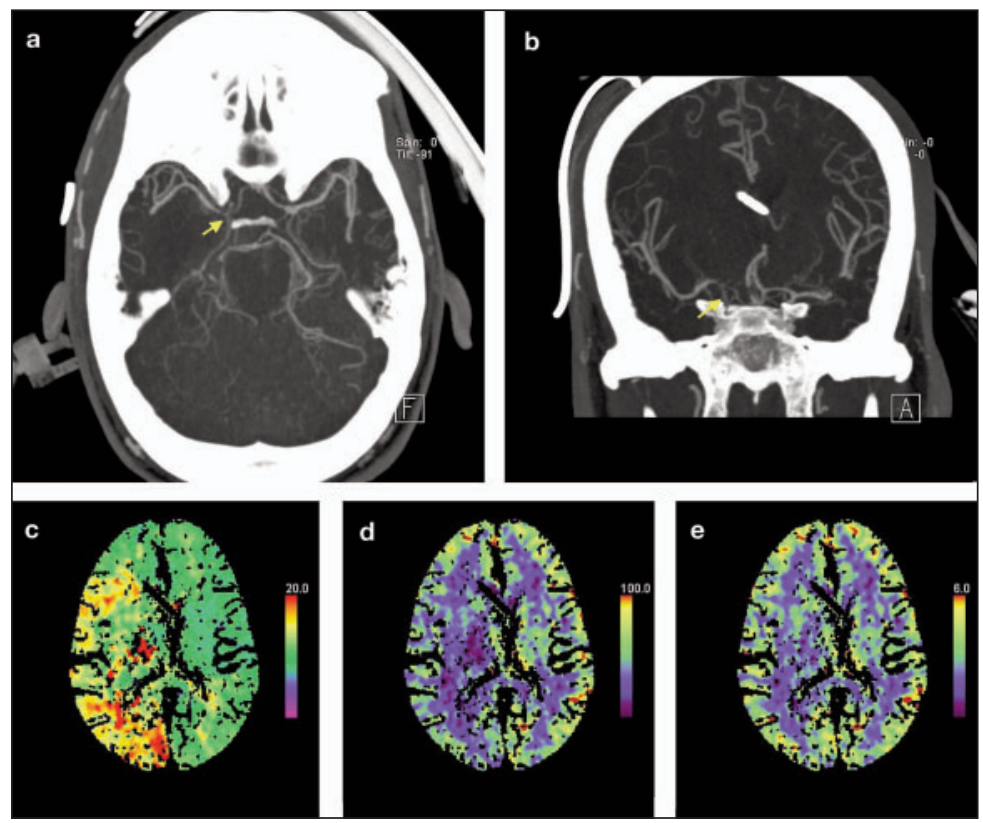

Figure 1: 48-year-old male, 9 days post $S A H$, had a decrease in his level of consciousness, which did not respond to hypertensive and hypervolumic treatment. Axial (a) and coronal (b) maximum intenstity projection $(M I P)$ demonstrates severe right distal ICA vasospasm (yellow arrow) extending into proximal MI and Al segments. TTP color map (c) demonstrates increased time to peak in the right cerebral hemisphere alongwith with decreased $C B F(d)$ in the same distribution. The $C B V$ is preserved (e). The patient underwent successful angioplasty for proximal vasospasm and intra-arterial infusion with milrinone for distal vasospasm.

The pooled estimates for distal vasospasm were not statistically different with $85.5 \%$ sensitivity and $92.3 \%$ specificity. ${ }^{15}$ Yoon et $a l$ also specifically evaluated proximal and distal CV and found no statistically significant difference in sensitivity of specificity between proximal or distal CV. ${ }^{12}$ A more recent study by Shankar et al once again demonstrated high sensitivity and specificity using the CTA to diagnose CV. The sensitivity and specificity were found to be slightly lower in the assessment of peripheral $\mathrm{CV}$ versus central CV. The sensitivity and specificity for central CV was $91-92 \%$ and $73-90 \%$ respectively. This dropped to 82 - $90 \%$ sensitivity and $50-69 \%$ specificity in the assessment of peripheral vasospasm. ${ }^{16}$

The majority of the literature suggests that CTA has a high sensitivity and specificity in the diagnosis of CV. There is some evidence that CTA may be less accurate in the assessment of peripheral vessels compared with central vessels.

Dankbaar et al specifically looked at the diagnosis of DCI with different CT modalities and showed that CTA had sensitivity and specificity of $64 \%$ and $50 \%$ respectively. ${ }^{17}$ This study was not a comparison with DSA as a gold standard, but used the clinical diagnosis of DCI as the gold standard. This data does not contradict the higher sensitivities and specificities previously reported, it just reaffirms that not all $\mathrm{CV}$ will result in DCI.

\section{CT Perfusion in Vasospasm}

Computed tomogram perfusion is often used in the assessment of embolic stroke. It involves CT imaging repeatedly obtained through a region of the brain during the administration of a small bolus of intravenous contrast. After administration of intravenous contrast, there is a transient increase in the attenuation of the brain parenchyma. This is proportional to the amount of contrast material within the brain parenchyma and this data can be plotted versus time for arterial, venous and parenchymal regions of interest. Post-processing can provide several different parameters with this data. The most commonly acquired data are cerebral blood flow (CBF), cerebral blood volume $(\mathrm{CBV})$, mean transit time (MTT) and time to peak (TTP). The CT perfusion does not directly visualize areas of arterial narrowing as CTA and DSA do, but instead detects areas of altered perfusion, which will often be due to $\mathrm{CV}$ in A-SAH patients. Figures $1 \mathrm{c}$ and $1 \mathrm{~d}$ demonstrate abnormal TTP and CBF related to $\mathrm{CV}$.

There have been several studies assessing the use of CTP in the evaluation of $\mathrm{CV}$ and/or DCI after A-SAH. Although CTP does not directly visualize cerebral arteries, several studies have compared CTP with DSA to determine if perfusion abnormalities are accurate in the diagnosis of $\mathrm{CV}$. This data is summarized in the Table. Several groups have evaluated CTP for the diagnosis of DCI at the time of clinical worsening and some have evaluated the use of CTP to predict which patients are at increased risk of progressing to DCI, even if they do not have clinical worsening at the time of the CTP study.

A study by Moftakhar et al compared CTP with DSA and found a $91 \%$ concordance rate in predicting the presence or absence of CV. ${ }^{18}$ Binaghi et al also compared CTP with DSA to diagnose $\mathrm{CV}$ and showed a high sensitivity and specificity of $90 \%$ and $100 \%$ respectively for severe vasospasm, but only $20 \%$ sensitivity and $100 \%$ specificity for mild - moderate vasospasm. ${ }^{13}$ A meta-analysis by Greenberg et al that compared CTP with DSA showed $74.1 \%$ sensitivity and $93 \%$ specificity for CTP in diagnosing CV. ${ }^{15}$

Dankbaar et al assessed CTP for the diagnosis of DCI and showed an $84 \%$ sensitivity and $79 \%$ specificity. ${ }^{17}$ A study by Killeen et al compared CTP with DSA in diagnosing DCI. They found similar characteristics for the two tests with sensitivity and specificity of $80 \%$ and $67 \%$ respectively for CTP compared with $73 \%$ and $75 \%$ respectively for DSA. They also found a 
significant difference in CBF values between DCI and non-DCI patients with $29.4 \mathrm{~mL} / 100 \mathrm{~g} / \mathrm{min}$ for the DCI group and 40.5 $\mathrm{mL} / 100 \mathrm{~g} / \mathrm{min}$ for the non-DCI group. ${ }^{19}$ Another study by Dankaar et al examined threshold values for CTP in diagnosing DCI and found a significant difference in CBF, MTT and perfusion asymmetry between patients with DCI and those without. An MTT threshold of 5.9 seconds optimized sensitivity and specificity, however this cutoff time will vary with each CT scanner and CTP protocol. ${ }^{20}$ Lefournier et al were not able to determine a threshold value for MTT that could predict patients with vasospasm. ${ }^{21}$ Sanelli et al evaluated quantitative CTP for the diagnosis of DCI and were able to determine a threshold level of $35 \mathrm{~mL} / 100 \mathrm{~g} / \mathrm{min}$ for CBF had a sensitivity of $90 \%$ and specificity of $68 \%$ while a threshold of 5.5 seconds for MTT had a sensitivity of $73 \%$ and specificity of $79 \%$. They also performed a subanalysis for the diagnosis of $\mathrm{CV}$ on the patients who underwent DSA. They found similar thresholds of 36.5 $\mathrm{mL} / 100 \mathrm{~g} / \mathrm{min}$ for CBF had a sensitivity of $95 \%$ and specificity of $70 \%$, and 5.4 seconds for MTT had a sensitivity of $78 \%$ and specificity of $70 \% .{ }^{22}$ Wintermark et al compared CTP with DSA in an attempt to determine threshold values for CBF, CBV and MTT for the diagnosis of CV. They showed high sensitivity of 95.1\% using an MTT threshold value of greater than 6.4 seconds. ${ }^{11}$

In terms of predicting which patients will proceed to DCI, van der Schaaf et al performed CTP in patients within 72 hours of aneurysm hemorrhage and assessed the CBF ratio (a ratio of $\mathrm{CBF}$ values from the same region in each hemisphere) as a predictor for the development of DCI. They showed that CBF ratio in the acute phase was an independent predictor for the development of DCI. ${ }^{23}$ Pham et al assessed the predictive value of CTP for later acquiring a secondary cerebral infarction and found that visual assessment of TTP maps were most sensitive at $93 \%$, but only demonstrated $67 \%$ specificity. ${ }^{24}$ Dankbaar et al showed that patients that are going to progress to DCI already have abnormal CBF and MTT values before any focal clinical worsening. ${ }^{25}$ Sanelli et al performed CTP early after A-SAH and found that $\mathrm{CBF}$ reduction and MTT prolongation demonstrated high specificity for later development of $\mathrm{CV}$. The standard to determine if $\mathrm{CV}$ developed was a multi-stage process with DSA at the primary level. A CBF threshold value of 24-25 $\mathrm{mL} / 100 \mathrm{~g} / \mathrm{min}$ showed $91 \%$ specificity for patients going on to have $\mathrm{CV} .{ }^{26}$ Sanelli et al were also able to determine that CTP had a sensitivity of $78 \%$ and specificity of $66 \%$ for patients who would develop permanent neurologic deficits and sensitivity of $88 \%$ and specificity of $59 \%$ for patients who would later develop an infarct. Their quantitative analysis showed that a CBF of 30.5 $\mathrm{mL} / 100 \mathrm{~g} / \mathrm{min}$ and an MTT of 5.0 seconds were optimal threshold values. ${ }^{27}$

There are case reports in patients with $\mathrm{CV}$ that demonstrate improvement or resolution of CTP abnormalities after intervention, indicating CTP may be an accurate way to assess intervention success. Majoie et al. report a case of using CTP before and after intraarterial intervention with abnormal CBV and MTT prior to intervention which then normalized on the post procedure CTP study. ${ }^{28} \mathrm{~A}$ similar result was reported by Shankar et al. ${ }^{29}$

The literature generally suggests that perfusion abnormalities on CTP are accurate in the diagnosis of $\mathrm{CV}$ when compared with
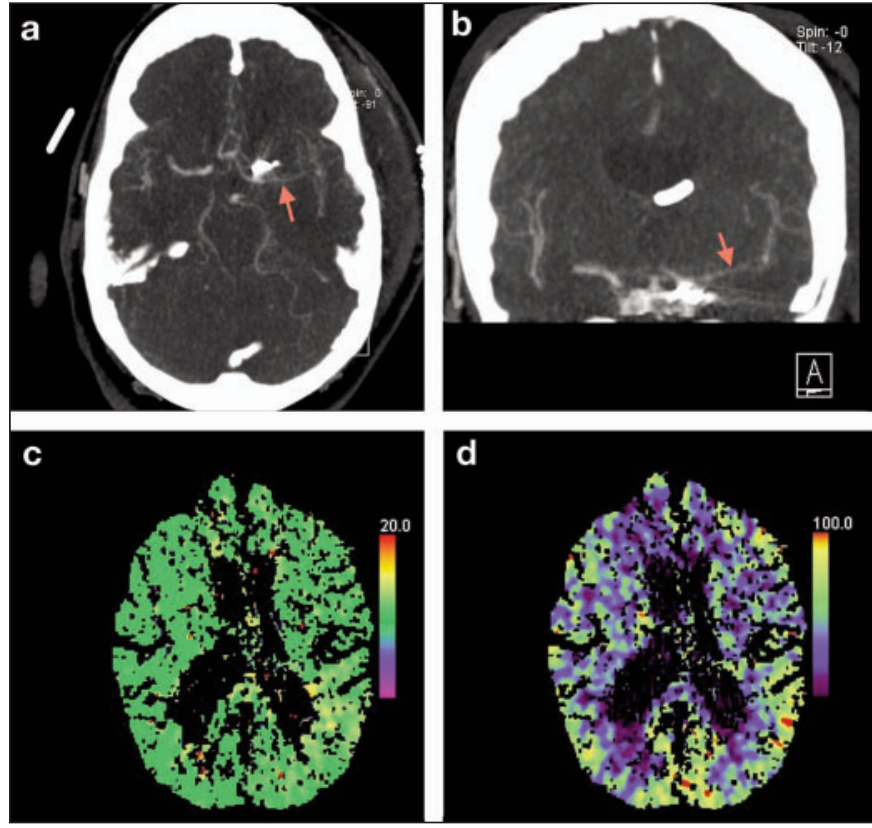

Figure 2: 54-year-old female, 4 days post SAH, developed new left pronator drift and had decrease in Glasgow Coma Scale. Axial (a) and coronal (b) MIP CTA demonstrates severe left MCA vasospasm (red arrow). Surprisingly, CTP demonstrated preserved TTP (c) and CBF (d). $C B V$ (not shown) was also normal.

DSA. It also performs well in the diagnosis of DCI. There is also evidence that CTP abnormalities present before clinical worsening signify an increased risk of progressing to DCI.

\section{CTA and CTP in Treatment Decisions}

Due to the fact that not all radiographically evident vasospasm will result in clinical deterioration and/or development of an infarct, the decision whether or not to treat vasospasm is often a difficult one. The fact that many of these patients will be in an ICU setting and sedated makes evaluation of clinical symptoms difficult and makes treatment decisions even more difficult. Several of the studies in the preceding sections specifically evaluated CTA and/or CTP with respect to treatment decisions. Wintermark et al performed a sub-analysis on patients who required treatment for $\mathrm{CV}$ and showed that CTA only had a sensitivity of $53.3 \%$ for patients requiring treatment, but maintained a high specificity of $96.3 \%$. They also did the same with CTP data and showed that an abnormal MTT with cutoff of greater than 7.6 seconds had a sensitivity of $70.0 \%$ and specificity of $87.5 \%$ and that an abnormal CBF with cutoff of less than $39.3 \mathrm{ml} / 100 \mathrm{~g} / \mathrm{min}$ had a sensitivity of $61.7 \%$ and very high specificity of $98.2 \% .{ }^{11}$ Shankar et al compared a treatment recommendation based on CTA findings with actual treatment received and found good agreement for patients who would receive endovascular treatment, but not for patients who would only receive medical treatment (triple $\mathrm{H}$ therapy). ${ }^{16}$ Binaghi et al found that the addition of CTP data "strongly influenced" the decision to treat in 9/27 patients when CTP was added to CTA. ${ }^{13}$

There is not a large amount of published data with respect to 


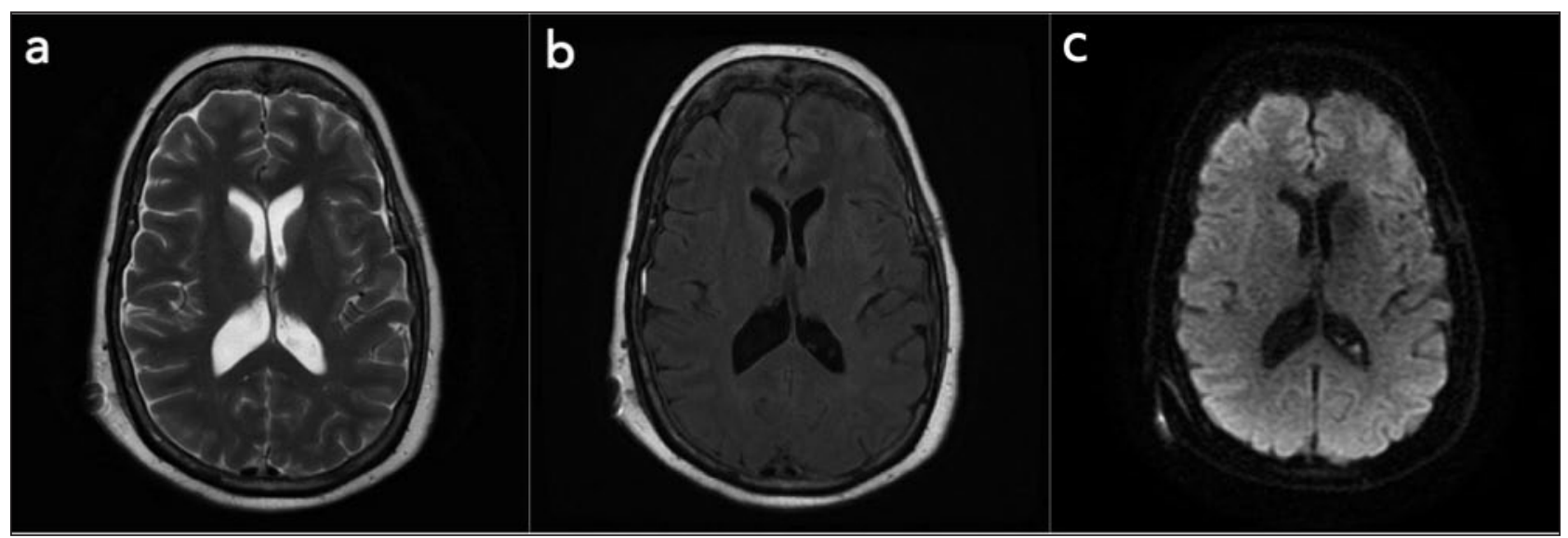

Figure 3: The same patient shown in figure 2 made a complete recovery clinically and had a follow-up MRI done 3 weeks later. Axial T2 (a), FLAIR (b) and DWI (c) sequnces show no evidence of stroke.

using results from CTA or CTP in terms of how it affects treatment. There is some evidence that CTP may provide more information than CTA alone in terms of guiding treatment decisions. CTP with abnormal MTT and CBF values has higher sensitivity for patients who require endovascular treatment compared with CTA and is still able to maintain a high specificity. It is likely that patients with abnormal CTA and CTP would require endovascular treatment, but it is not clear whether patients with abnormal CTA, but normal CTP also require endovascular treatment. Figure 2 demonstrates evidence of $\mathrm{CV}$ on CTA without evidence of CTP abnormality in a patient who recovered without endovascular treatment and had no stroke on follow-up MRI (Figure 3).

The CTP parameters are dependant on various hemodynamic parameters and hence are dynamic. It is important to have a protocol for regular monitoring of these changes. Outside the ICU setting, this monitoring is usually done using frequent clinical examinations. For patients in ICU, where clinical examination is extremely limited, these imaging tools play a significant role in management decisions. Future studies are required to come up with an acceptable guideline for perhaps a combination of both CTA and CTP in these patients.

\section{Limitations of CTA and CTP}

A limitation of CTA and CTP is radiation exposure. However, compared with DSA, the radiation dose from CTA and CTP is generally lower. A recent study by Manninen et al showed that the effective radiation dose of CTA in the assessment of cerebral vessels was approximately $20 \%$ that of DSA. ${ }^{30}$ In terms of CTP, there are multiple factors that can be altered to attempt to minimize the radiation exposure, including tube voltage, ${ }^{31}$ tube current, ${ }^{32}$ scan duration, ${ }^{33}$ pitch and sampling interval. ${ }^{34}$ Another limitation of CTA and CTP is the placement of an IV catheter and the administration of iodinated contrast material. There are multiple studies that have shown that injection of contrast media for CTA and CTP in acute stroke setting does not cause renal failure (REF). However in cases of $\mathrm{CV}$, where multiple CTP/CTA studies may be required in any given patient, the effect is yet to be reported. In terms of CTP, there is also limitation with respect to quantitative analysis. $\mathrm{CBF}, \mathrm{CBV}, \mathrm{MTT}$ and TTP values will vary between based on equipment and technique, therefore quantitative threshold values are not immediately transferable between different institutions.

Until recently, CT scanners were unable to provide whole brain coverage with CTP protocols. The majority of the published literature using CTP in $\mathrm{CV}$ has been done using older 16 or 64 slice CT scanners, which only provide a thin slab of brain coverage. With modern CT scanners, this is no longer the case, and some scanners are able to provide whole brain coverage using a CTP protocol. ${ }^{29}$

\section{Conclusions}

Cerebral vasospasm is an important and potentially devastating complication after A-SAH. The ultimate goal is the early diagnosis of patients that will progress to DCI in order to enact treatment and halt the progression.

The gold standard for the diagnosis of $\mathrm{CV}$ is considered to be DSA, however the non-invasive technique of CTA and functional technique of CTP demonstrate significantly high sensitivity and specificity both for the diagnosis of $\mathrm{CV}$ and prediction of progression to DCI. Functional information on CTP is more promising for guiding the treatment decision. Due to significant radiation dose, there is no consensus on what should be the proper monitoring protocol for CV using CTA and CTP.

\section{REFERENCES}

1. Ingall $\mathrm{T}$, Asplund $\mathrm{K}$, Mähönen $\mathrm{M}$, Bonita R. A multinational comparison of subarachnoid hemorrhage epidemiology in the WHO MONICA stroke study. Stroke. 2000;31:1054-61.

2. Velat GJ, Kimball MM, Mocco JD, Hoh BL. Vasospasm after aneurysmal subarachnoid hemorrhage: review of randomized controlled trials and meta-analyses in the literature. World Neurosurg. 2011;76:446-54.

3. Kassell NF, Sasaki T, Colohan AR, Nazar G. Cerebral vasospasm following aneurysmal subarachnoid hemorrhage. Stroke. 1985; 16:562-72.

4. Kimball MM, Velat GJ, Hoh BL. Critical care guidelines on the endovascular management of cerebral vasospasm. Neurocrit Care. 2011;15:336-41. 
5. Zabramski JM, Spetzler RF, Bonstelle C. Chronic cerebral vasospasm: effect of volume and timing of hemorrhage in a canine model. Neurosurgery. 1986;18:1-6.

6. Fisher CM, Kistler JP, Davis JM. Relation of cerebral vasospasm to subarachnoid hemorrhage visualized by computerized tomographic scanning. Neurosurgery. 1980;6:1-9.

7. Kolias AG, Sen J, Belli A. Pathogenesis of cerebral vasospasm following aneurysmal subarachnoid hemorrhage: putative mechanisms and novel approaches. J Neurosci Res. 2009;87: $1-11$.

8. Vergouwen MDI, Vermeulen M, van Gijn J, et al. Definition of delayed cerebral ischemia after aneurysmal subarachnoid hemorrhage as an outcome event in clinical trials and observational studies: proposal of a multidisciplinary research group. Stroke. 2010;41:2391-5.

9. Anderson GB, Ashforth R, Steinke DE, Findlay JM. CT angiography for the detection of cerebral vasospasm in patients with acute subarachnoid hemorrhage. AJNR Am J Neuroradiol. 2000;21:1011-15.

10. Goldsher D, Shreiber R, Shik V, Tavor Y, Soustiel JF. Role of multisection CT angiography in the evaluation of vertebrobasilar vasospasm in patients with subarachnoid hemorrhage. AJNR Am J Neuroradiol. 2004;25:1493-8.

11. Wintermark M, Ko NU, Smith WS, Liu S, Higashida RT, Dillon WP. Vasospasm after subarachnoid hemorrhage: utility of perfusion CT and CT angiography on diagnosis and management. AJNR Am J Neuroradiol. 2006;27:26-34.

12. Yoon DY, Choi CS, Kim KH, Cho B-M. Multidetector-row CT angiography of cerebral vasospasm after aneurysmal subarachnoid hemorrhage: comparison of volume-rendered images and digital subtraction angiography. AJNR Am J Neuroradiol. 2006;27:370-7.

13. Binaghi S, Colleoni ML, Maeder P, et al. CT angiography and perfusion $\mathrm{CT}$ in cerebral vasospasm after subarachnoid hemorrhage. AJNR Am J Neuroradiol. 2007;28:750-8.

14. Chaudhary SR, Ko N, Dillon WP, et al. Prospective evaluation of multidetector-row CT angiography for the diagnosis of vasospasm following subarachnoid hemorrhage: a comparison with digital subtraction angiography. Cerebrovasc Dis. 2008;25: $144-50$.

15. Greenberg ED, Gold R, Reichman M, et al. Diagnostic accuracy of CT angiography and CT perfusion for cerebral vasospasm: a meta-analysis. AJNR Am J Neuroradiol. 2010;31:1853-60.

16. Shankar JJS, Tan IYL, Krings T, Terbrugge K, Agid R. CT angiography for evaluation of cerebral vasospasm following acute subarachnoid haemorrhage. Neuroradiology. 2012;54: 197-203.

17. Dankbaar JW, de Rooij NK, Velthuis BK, Frijns CJM, Rinkel GJE, van der Schaaf IC. Diagnosing delayed cerebral ischemia with different CT modalities in patients with subarachnoid hemorrhage with clinical deterioration. Stroke. 2009;40:3493-8.

18. Moftakhar R, Rowley HA, Turk A, et al. Utility of computed tomography perfusion in detection of cerebral vasospasm in patients with subarachnoid hemorrhage. Neurosurg Focus. 2006; 21:E6.
19. Killeen RP, Mushlin AI, Johnson CE, et al. Comparison of CT perfusion and digital subtraction angiography in the evaluation of delayed cerebral ischemia. Acad Radiol. 2011;18:1094-100.

20. Dankbaar JW, de Rooij NK, Rijsdijk M, et al. Diagnostic threshold values of cerebral perfusion measured with computed tomography for delayed cerebral ischemia after aneurysmal subarachnoid hemorrhage. Stroke. 2010;41:1927-32.

21. Lefournier V, Krainik A, Gory B, et al. Perfusion CT to quantify the cerebral vasospasm following subarachnoid hemorrhage. J Neuroradiol. 2010;37:284-91.

22. Sanelli PC, Ugorec I, Johnson CE, et al. Using quantitative CT perfusion for evaluation of delayed cerebral ischemia following aneurysmal subarachnoid hemorrhage. AJNR Am J Neuroradiol. 2011;32:2047-53.

23. van der Schaaf I, Wermer MJ, van der Graaf Y, Hoff RG, Rinkel GJE, Velthuis BK. CT after subarachnoid hemorrhage: relation of cerebral perfusion to delayed cerebral ischemia. Neurology. 2006;66:1533-8.

24. Pham M, Johnson A, Bartsch AJ, et al. CT perfusion predicts secondary cerebral infarction after aneurysmal subarachnoid hemorrhage. Neurology. 2007;69:762-5.

25. Dankbaar JW, de Rooij NK, Smit EJ, et al. Changes in cerebral perfusion around the time of delayed cerebral ischemia in subarachnoid hemorrhage patients. Cerebrovasc Dis. 2011;32: 133-40.

26. Sanelli PC, Jou A, Gold R, et al. Using CT perfusion during the early baseline period in aneurysmal subarachnoid hemorrhage to assess for development of vasospasm. Neuroradiology. 2011;53: 425-34.

27. Sanelli PC, Anumula N, Johnson CE, et al. Evaluating CT perfusion using outcome measures of delayed cerebral ischemia in aneurysmal subarachnoid hemorrhage. AJNR Am J Neuroradiol. 2013;34:292-8

28. Majoie CBLM, van Boven LJ, van de Beek D, Venema HW, van Rooij WJ. Perfusion CT to evaluate the effect of transluminal angioplasty on cerebral perfusion in the treatment of vasospasm after subarachnoid hemorrhage. Neurocrit Care. 2007;6:40-4.

29. Shankar JJS, Lum C. Whole brain CT perfusion on a 320-slice CT scanner. Indian J Radiol Imaging. 2011;21:209-14.

30. Manninen A-L, Isokangas J-M, Karttunen A, Siniluoto T, Nieminen MT. A comparison of radiation exposure between diagnostic CTA and DSA examinations of cerebral and cervicocerebral vessels. AJNR Am J Neuroradiol. 2012;33:2038-42.

31. Wintermark M, Maeder P, Verdun FR, et al. Using $80 \mathrm{kVp}$ versus $120 \mathrm{kVp}$ in perfusion CT measurement of regional cerebral blood flow. AJNR Am J Neuroradiol. 2000;21:1881-4.

32. Murase K, Nanjo T, Ii S, et al. Effect of x-ray tube current on the accuracy of cerebral perfusion parameters obtained by CT perfusion studies. Phys Med Biol. 2005;50:5019-29.

33. Hirata M, Sugawara Y, Murase K, Miki H, Mochizuki T. Evaluation of optimal scan duration and end time in cerebral CT perfusion study. Radiat Med. 2005;23:351-63.

34. Shankar JJS, Lum C, Sharma M. Whole-brain perfusion imaging with 320-MDCT scanner: Reducing radiation dose by increasing sampling interval. AJR Am J Roentgenol. 2010;195:1183-6. 